Military Technical College

Kobry Elkobbah,

Cairo, Egypt

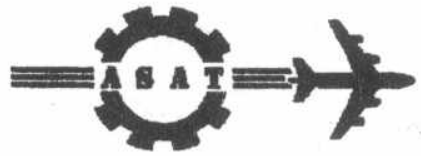

$8^{\text {th }}$ International Conference

on Aerospace Sciences \&

Aviation Technology

\title{
ANALYTIC SENSITIVITIES IN COMPOSITE WING-SHAPE YNTHESIS PART TWO: PANEL FLUTTER CONSTRAINTS
}

\author{
M.M. ELNOMROSSY*, M.A. MEHANNA**
}

\begin{abstract}
The equivalent plate modeling formulation of composite wings, described in Part One, is extended in this paper to include the explicit analytic expressions for terms of the mass and aerodynamic matrices. Analytic sensitivities of the mass and aerodynamic matrices with respect to panel shape, thickness and fiber orientations are derived. Before proceeding in the study of analytic sensitivities using the derived expressions, the accuracy of its panel flutter analysis was numerically tested for simply supported panels of trapezoidal and skewed shape, of isotropic and composite materials and variable fiber orientations. The nondimensional critical dynamic pressure and critical frequency of flutter are compared with the results of finite element flutter analysis of composite skew panels by Chowdary, T.V.R. and al given in "Computers and Structures", (1996). The correlation of the results is good for moderate in-plane loading. Accuracy of the approximations is studied for the integration with nonlinear programrning/approximation concept aeroelastic design synthesis methodology.
\end{abstract}

\section{KEY WORDS}

composite wing, equivalent plate, flutter, analytic sensitivity analysis, mass matrix, aerodynamic force matrices, finite element method.

\section{NOMENCLATURE}

[A $\left.A_{\text {stif }}\right]$ : aerodynamic stiffness matrix

[A damp] :aerodynamic damping matrix

[C] : damping matrix

DV: design variable

$\left[F_{1}\right],\left[F_{2}\right],\left[F_{3}\right]$ : matrices containing admissible functions and their derivatives

\footnotetext{
* Staff Member, Aerospace Department, Military Technical College, Cairo

** Engineer, Egyptian Armed Forces.
} 
$f_{i}(x, y)$ : admissible functions

$h(x, y)$ : total thickness of panel

$I_{T R}$ : integral of a simple polynomial term over the trapezoidal panel area

$[\mathrm{K}]$ : stiffness matrix

$\left[K_{G}\right]$ : geometric stiffness matrix

$\left[\mathrm{K}^{\star}\right]$ : generalized stiffness matrix including structural and aerodynamic effects

[M]: mass matrix

$M_{\alpha}$ : flight Mach number

$m, n$ : powers of $x$ and $y$ in a polynomial

$\mathrm{m}_{\mathrm{k},}^{t} \mathrm{n}_{\mathrm{k}}^{\mathrm{t}}$ : powers of $\mathrm{x}$ and $\mathrm{y}$ in polynomial series for thickness of layer $\mathrm{I}$

$m^{w \prime}{ }_{k}, n^{w}{ }_{k}$ : powers of $x$ and $y$ in Ritz functions

$m^{v}, n_{j}, n_{j} u_{j}$ powers of $x$ and $y$ in polynomial terms of $F_{B}(x, y)$

$\mathrm{N}_{\mathrm{i}}$ : number of terms in the thickness polynomial foe layer I

$N_{L}$ : number of layers

$N_{x}, N_{y}, N_{x y}$ in-plane loads per unit length

$\Delta \mathrm{p}$ : pressure difference distribution

$\left\{Q_{A}\right\}$ : generalized aerodynamic fórce matrix

$\left[Q_{\text {stiff }}\right]$ : Mach independent aerodynamic stiffness matrix

$\left[Q_{\text {damp }}\right]$ : Mach independent aerodynamic damping matrix

$q_{D}$ : flight dynamic pressure

$q_{\text {cititcal }}$ : critical dynamic pressure

$\{q\}$ : vector of generalized displacements for panel

$\mathrm{T}_{k}$ : the ith coefficient in the polynomial series for thickness of the kth layer

$U_{0}$ : flight speed

$w(x, y)$ : panel out-of-plane displacements

$\Lambda, \Omega$ : normalized dynamic pressure and frequency

$\lambda$ : eigenvalues

$\xi$ : direction of incoming flow

$\rho_{\mathrm{m}}$ : plate material density

$\rho_{\alpha}$ : air density

$\{\phi\}$ : right eigenvector

$\{\psi\}$ : left eigenvector

\section{Subscripts}
A : aft (rear)
crit : critical dynamic pressure
$F$ : front
flutter: flutter dynamic pressure
$L$ : left
$\mathrm{R}$ : right 


\section{INTRODUCTION}

An extensive work and consequently substantial knowledge and experience have been accumulated over the past four decades regarding the aeroelasticity of panels in supersonic flow, [1] and [2]. Different numerical techniques have been used together with exact solutions including Galerkin, Rayleigh-Ritz and finite element techniques, [3], [4] and [5]. As an aeroelastic research problem, it includes linear and nonlinear stability and dynamic response, dynamics of systems with random parameters,[6], and interactions between static and dynamic instabilities in the presence of in-plane loads and thermal effects,[7] and [8]. Panel flutter has been used to study applications of composite materials, [9] and [10], and transverse shear effects,[11]. Most of the studies, however, are confined to rectangular panels. Solution techniques and results for quadrilateral and trapezoidal panels have been discussed in only a small number of articles, [12], [13] and [14].

Skin panels in typical aircraft wing structures are very often trapezoidal in shape. Moreover, during the shape optimization of such wing structures, internal spars, ribs and stiffeners may move to form trapezoidal skin panels, and these panels may change shape during optimization in addition to changing thickness and material properties. The capability to efficiently evaluate the aeroelastic stability of trapezoidal skin panels under combined in-plane loads, as well as sensitivities with respect to sizing, material and shape design variables, constitute an important building block in an overall structural/aeroelastic optimization capability.

The optimization of panels, subject to aeroelastic constraints, has been studied in cases involving isotropic and composite structures,[15], [16], [17] and [18]. These studies are limited to the treatment of an isolated rectangular panel, excluding its interaction with the structure containing it. In the effort to develop effective aeroservoelastic synthesis techniques for stressed skin aerospace structures, the area of airframe shape optimization becomes important, because of the need to make rigorous design optimization available to the designer at an early stage of the design process, where overall shape of the aircraft is still evolving. Analytic shape sensitivities have been developed for wing box structural modeling,[19], integrated wing box/panel buckling analysis in part one of this paper and unsteady aerodynamics in both subsonic and supersonic flight,[20]. This second part of the paper focuses on the integrated wing box/panel flutter analysis and sensitivity problem with emphasis on the needs of planform shape optimization.

This paper uses the panel modeling and the use of simple polynomials as admissible functions in the Ritz analysis given in part one of the paper. In part two of the paper, it is shown how this formulation leads to the expression of mass and aeroclynamic matrix terms as linear combinations of members of a single table of area integrals, obtained explicitly over the trapezoidal area of the panel. Analytic sensitivities with respect to thickness, fiber orientation and panel shape are derived. Test cases and results conclude this article. 


\section{MASS MATRIX}

When the kinetic energy of the panel in transverse motion is expressed in terms of the Ritz function, equation 9150 of part one of this paper, the mass matrix is found to be:

$$
[\mathrm{M}]=\iint_{\text {aera }} \rho_{\mathrm{m}} \mathrm{h}\left[\mathrm{F}_{1}\right]^{\mathrm{T}}\left[\mathrm{F}_{1}\right] \mathrm{dxdy}
$$

where $\rho_{m}$ is the material density, $h(x, y)$ is the panel thickness, and the matrix $\left[F_{1}\right]$ contains the admissible functions, and is, thus, also a function of $x$ and $y$. The rs elernent of the mass matrix is given by:

$$
M_{r s}=\iint_{\text {area }} \rho r_{M} h f_{r} f_{s} d x d y
$$

The thickness series used for the different skin layers, equations (2) and (3) of part one of this paper, shows that if the thickness distributions of all layers are expressed by complete polynomials of the same order, then all layers will have the same number of terms $N_{L}$ and the same powers $m_{k}^{t}$ and $n_{k}^{t}$ in their respective thickness series. A new vector of thickness coefficients $T^{*}{ }_{k}$ is defined to add all $T_{k}^{i}$ terms associated with the same powers of $x$ and $y$.

$$
\overline{\mathrm{T}}_{\mathrm{k}}=\sum_{\mathrm{i}=1}^{\mathrm{N}_{\mathrm{L}}} \mathrm{T}_{\mathrm{k}}^{\mathrm{i}}
$$

Now, the total panel thickness is expressed as:

$$
h(x, y)=\sum_{k=1}^{N_{t}} \bar{T}_{k} x^{m_{k}^{\prime}} y^{n_{k}^{i}}
$$

Substituting into Eq.(2) leads to:

$$
M_{r s}=\rho_{m} \sum_{k=1}^{N_{k}} \bar{T}_{k} \iint_{\text {area }} f_{r} r_{s} x^{m_{k}^{t}} y^{n_{k}^{i}} d x d y
$$

When the polynomial admissible Ritz functions are used in Eq.(5), then the explicit form of mass matrix elements is obtained:

$$
M_{r s}=\rho_{m} \sum_{k=1}^{N_{k}} \sum_{i=1}^{3} \sum_{j=1}^{6} \sum_{i=1}^{3} \sum_{i=1}^{6} \bar{T}_{k} U_{i} V_{j} U_{i j} V_{i j} \iint_{\text {area }} x^{m} y^{n} d x d y
$$

where the powers $m$ and $n$ are: 


$$
\begin{aligned}
& m=m_{j}^{v}+m_{i j}^{v}+m_{r}^{w}+m_{s}^{w}+m_{k}^{t} \\
& n=n_{i}^{u}+n_{i j}^{u}+n_{j}^{v}+n_{i j}^{v}+n_{r}^{w}+n_{s}^{w}+n_{k}^{t}
\end{aligned}
$$

Note that all of the elements of the mass matrix are linear combinations of area integral $I_{T R}(m, n)$ of simple polynomials over the trapezoidal panel. The mass matrix is dependent on the material density, the thickness terms $T_{k}$ and the panel shape variables (through $U_{1}$ and $V_{j}$ as given by equation (11) of part one of this paper).

\section{AERODYNAMIC FORCE MATRICES}

When the virtual work due to surface pressure difference distribution $\Delta p(x, y)$ is expressed in terms of the Ritz functions, equations (13) and (14) of part one of this paper, the vector of generalized aerodynamic forces is obtained, [6] and [7].

$$
\left\{Q_{A}\right\}=\iint_{\text {area }}\left[F_{1}\right]^{\top} \Delta p(x, y) d x d y
$$

First-order linear piston theory approximates $\Delta p(x, y)$ by:

$$
\Delta p(x, y)=\frac{\rho_{\infty} U_{\infty}^{2}}{\sqrt{M_{\infty}^{2}-1}}\left\{w_{, x \xi}+\frac{M_{\infty}^{2}-2}{M_{\infty}^{2}-1} \frac{1}{U_{\infty}} w_{, t}\right\}
$$

where $\rho_{m}, U_{\infty}$ and $M_{\infty}$ are the free stream density, velocity and Mach number, respectively, and $\xi$ is the flow direction.

Panels are aligned so that their left and right sides are parallel to the $x$-axis. When the in-coming flow is yawed with respect to the $x$ axis, creating an angle $\varphi$ with the $x$ axis, then the slope of panel deformation along the flow direction $\xi$ is given by:

$$
\frac{\partial}{\partial x}=\cos \phi \frac{\partial}{\partial x}+\sin \phi \frac{\partial}{\partial y}
$$

The pressure difference according to first-order piston theory can now be rewritten as:

where

$$
\Delta p(x, y, t)=P_{\xi} \cos \phi w_{x}+P_{\xi} \sin \phi w_{, y}+P_{t} w_{, t}
$$

$$
\begin{aligned}
& P_{\xi}=\frac{\rho_{\infty} U_{\infty}^{2}}{\sqrt{M_{\infty}^{2}-1}} \\
& P_{t}=P_{\xi} \frac{M_{\infty}^{2}-2}{M_{\infty}^{2}-1} \frac{1}{U_{\infty}}
\end{aligned}
$$


Equation (15) of part one of this paper is now differentiated with respect to $x$ and then with respect to $y$ to give:

$$
\begin{aligned}
& w_{, x}(x, y)=\left[F_{1, x}\right]\{q\} \\
& w_{, y}(x, y)=\left[F_{1, y}\right]\{q\}
\end{aligned}
$$

Differentiating the same equation with respect to time leads to:

$$
w_{t}(x, y)=\left[F_{1}\right]\left\{q_{, t}\right\}
$$

Substituting Eqs. (14), (15) and (16) into Eq. (11) gives:

$$
\begin{gathered}
\left\{Q_{A}\right\}=P_{x} \operatorname{cosf} \iint_{\text {area }}\left[F_{1}\right]^{\top}\left[F_{1, x}\right]\{q\} d x d y+P_{x} \operatorname{sinf} \iint_{\text {area }}\left[F_{1}\right]^{\top}\left[F_{1, y}\right]\{q\} d x d y \\
+P_{t} \iint_{\text {area }}\left[F_{1}\right]^{\top}\left[F_{1}\right]\left\{q_{1}\right\} d x d y
\end{gathered}
$$

At this point, it is possible to define an aerodynamic stiffness matrix and an aerodynamic damping matrix:

$$
\left\{Q_{A}\right\}=P_{\xi}\left[Q_{\text {stiff }}\right]\{q\}+P_{t}\left[Q_{\text {damp }}\right]\left\{q_{, t}\right\}
$$

where the rs elements are given by:

$$
\begin{aligned}
& Q_{\text {stiff }_{n}}=\cos \phi \iint_{\text {area }} f_{r} f_{s, x} d x d y+\sin \phi \iint_{\text {area }} f_{r} f_{s, y} d x d y \\
& Q_{\text {damp }}=\iint_{\text {area }} f_{r} f_{s} d x d y
\end{aligned}
$$

where $f_{r}$ and $f_{s}$ are the $r^{\text {th }}$ and the $s^{\text {th }}$ admissible functions. The first derivatives of the adrnissible functions with respect to $x$ and $y$ are:

$$
\begin{aligned}
& f_{p, x}=\sum_{i=1}^{3} \sum_{j=1}^{6} U_{i} V_{j}\left(m_{j}^{v}+m_{p}^{w}\right) x^{\left(m_{j}^{v}+m_{p}^{w}-1\right)} y^{\left(n_{i}^{u}+n_{j}^{v}+n_{p}^{w}\right)} \\
& f_{p, y}=\sum_{i=1}^{3} \sum_{j=1}^{6} U_{i} V_{j}\left(n_{i}^{u}+n_{j}^{v}+n_{p}^{w}\right) x^{\left(m_{j}^{v}+m_{p}^{w}\right)} y^{\left(n_{i}^{u}+n_{j}^{v}+n_{p}^{w}-1\right)}
\end{aligned}
$$

Substituting these into Eqs.(19) and (20) leads to the final expression for the elements of the aerodynamic stiffness and damping matrices:

$$
\begin{aligned}
& Q_{\text {stiff }}=\cos \phi \sum_{i=1}^{3} \sum_{j=1}^{6} \sum_{i=1}^{3} \sum_{j=1}^{6} U_{i} V_{j} U_{i i} V_{i j}\left(m_{i j}^{v}+m_{s}^{w}\right) \iint_{\text {area }} x^{m-1} y^{n} d x d y \\
& +\sin \phi \sum_{i=1}^{3} \sum_{j=1}^{6} \sum_{i=1}^{3} \sum_{i j}^{6} U_{i} V_{j} \bigcup_{i i} V_{i j}\left(n_{i j}^{u}+n_{i j}^{v}+n_{s}^{w}\right) \iint_{\text {area }} x^{m} y^{n-1} d x d y \\
& Q_{\text {damp }}=\sum_{i=1}^{3} \sum_{j=1}^{6} \sum_{i=1}^{3} \sum_{j=1}^{6} U_{i} V_{j} U_{i j} V_{i j} \iint_{\text {area }} x^{m} y^{n} d x d y
\end{aligned}
$$

where the powers $m, n$ are given by: 


$$
\begin{aligned}
& m=m_{j}^{v}+m_{i j}^{v}+m_{r}^{w}+m_{s}^{w} \\
& n=n_{i}^{u}+n_{i j}^{u}+n_{j}^{v}+n_{j}^{v}+n_{r}^{w}+n_{s}^{w}
\end{aligned}
$$

The elements of the aerodynamic stiffness and damping matrices are, thus, linear combinations of the same area integrals as the mass, stiffness and geometric stiffness matrices. The aerodynamic stiffness matrix is dependent on the air stream yaw angle $\varphi$, the dynamic pressure and Mach number through $P_{\xi}$ and the panel shape variables through $U_{i}$ and $V_{j}$. The aerodynamic damping matrix is dependent on the dynamic pressure and Mach number through $\mathrm{P}_{\mathrm{t}}$, and on the panel shape variables through $U_{i}$ and $V_{j}$.

For aeroelastic stability analysis, it is convenient to express the aerodynamic matrices in the form:

$$
\begin{aligned}
& {\left[A_{\text {stiff }}\right]=\frac{1}{\sqrt{M_{\infty}^{2}-1}}\left[Q_{\text {stiff }}\right]} \\
& {\left[A_{\text {damp }}\right]=\frac{1}{\sqrt{M_{\infty}^{2}-1}} \frac{M_{\infty}^{2}-2}{M_{\infty}^{2}-1}\left[Q_{\text {damp }}\right]}
\end{aligned}
$$

which leads to an expression for the aerodynamic generalized forces in the form:

$$
\left\{Q_{A}\right\}=r_{\infty} U_{\infty}\left[A_{\text {damp }}\right]\left\{q_{1}\right\}+r_{\infty} U_{\infty}^{2}\left[A_{\text {stiff }}\right]\{q\}
$$

From Eqs. (23) and (24), the matrix [ $Q_{\text {stiff }}$ is skew-symmetric and the matrix $\left[Q_{\text {damp }}\right]$ is symmetric. Moreover, if the panel is made of a single material and has a constant thickness, then the aerodynamic damping matrix is proportional to the mass matrix.

\section{AEROELASTIC STABILITY ANALYSIS}

Using the expressions for the generalized aerodynamic forces, the equations of motion for the panel are:

$$
[M]\{\ddot{q}\}-r_{\infty} \cup_{\infty}\left[A_{\text {damp }}\right]\{\dot{q}\}+\left[[K]+\left[K_{G}\right]-r_{\infty} U_{\infty}^{2}\left[A_{\text {stiff }}\right]\right]\{q\}=\{0\}
$$

or

$$
[M]\{\ddot{q}\}+[C]\{\dot{q}\}+[\bar{K}]\{q\}=0
$$

Only aerodynamic damping is taken into account in the previous equations. If viscous structural damping is to be added, the matrix [C] should be modified,[21]. 
At a given altitude, Mach number, and corresponding speed, and for a given set of in-plane loads obtained from a wing box stress solution for the maneuvering airplane, the poles of the linear panel model can be found to determine whether the panel is aeroelastically stable or unstable. Laplace transforming Eq.(29) leads to:

$$
\left[[M] s^{2}+[C] s+[\bar{K}]\right]\{q(s)\}=\{0\}
$$

This quadratic $\mathrm{NxN}$ eigenvalue problem can be solved either directly or by an equivalent first-order generalized $2 \mathrm{~N} \times 2 \mathrm{~N}$ eigenproblem:

$$
s\left[\begin{array}{ll}
{[\mathrm{l}]} & {[0]} \\
{[0]} & {[\mathrm{M}]}
\end{array}\right]\left\{\begin{array}{l}
\mathrm{X}_{1} \\
\mathrm{x}_{2}
\end{array}\right\}=\left[\begin{array}{ll}
{[0]} & {[\mathrm{l}]} \\
-[\bar{K}] & {[0]}
\end{array}\right]\left\{\begin{array}{l}
\mathrm{x}_{1} \\
\mathrm{x}_{2}
\end{array}\right\}
$$

where

$$
\left\{x_{1}\right\}=\{q\} \quad \text { and } \quad\left\{x_{2}\right\}=s\{q\}
$$

The problem can be written in simpler notation as:

$$
[\overline{\mathrm{U}}]\{\phi\}=\lambda[\overline{\mathrm{V}}]\{\phi\}
$$

where $\lambda,\{\phi\}$ is an eigenvalue/right-eigenvector pair, and the matrices [ $\left.\mathrm{U}^{*}\right],\left[\mathrm{V}^{*}\right]$ are $2 \mathrm{~N} \times 2 \mathrm{~N}$ and e defined by Eqs.(28), (29) and (31).

Stability analysis at constant Mach number is carried out by gradually increasing the dynamic pressure and following the resulting movement of aeroelastic poles in the Laplace domain. Flutter instability (at the flutter dynamic pressure $q_{\text {fiutter }}$ ) corresponds to a pole crossing the imaginary axis from left to the right side of the s-plane at some nonzero frequency. Divergence instability corresponds to a pole crossing into the right-hand side of the s-plane on the real axis (with zero frequency). When no damping is included in the mathematical model (either structural or aerodynarnic), aeroelastic poles move on the imaginary axis as dynamic pressure is increased until a point $q_{\text {critical }}$, at which two poles coalesce and move off the imaginary axis to lie left and right of the imaginary axis,[22].

\section{ANALYTIC SENSITIVITIES}

Analytic sensitivities of stiffness and geometric stiffness matrices were presented in part one of this paper. Sensitivities with respect to thickness design variables and composite fiber angles have been obtained as well as sensitivities with respect to planform shape design variables. The key to obtaining analytic sensitivity expressions is the explicit closed-form nature of the expressions for stiffiness and geometric stiffness matrix terms. Analytic formulas are available for the area integrals,[19], and it shows that the shape derivatives of members of the table of 
integrals $I_{T R}(m, n)$ can be expressed in terms of order members of the same table of integrals. Thus, once the table of integrals is generiated (with $\mathrm{m}$ and $\mathrm{n}$ covering all powers required for the aeroelastic analysis of the panel) it can be used for both analysis and sensitivity.

\section{Mass matrix sensitivities}

1) Mass matrix sensitivities with respect to thickness design variables

The mass matrix is dependent on $T_{k}^{\prime}$, the sizing variable corresponding to the $k^{\text {th }}$ term of the $i^{\text {th }}$ layer, Eqs. (3) and (4). The derivative of a mass matrix term is given as:

$$
\frac{\partial M_{r s}}{\partial T_{k}^{i}}=\frac{\partial M_{r s}}{\partial \bar{T}_{k}} \cdot \frac{\partial \bar{T}_{k}}{\partial T_{k}^{i}}
$$

Based on the definition of $T^{*}{ }_{k}, E q .(3)$, then, for a given layer $i$ and thickness term $k$ :

$$
\frac{\partial \bar{T}_{k}}{\partial T_{k}^{i}}=1
$$

Substituting this back into Eq.(34) shows that differentiation with respect to the $k^{\text {th }}$ thickness term of any layer $i$ is the same as differentiating the mass matrix with respect to the $k^{\text {th }}$ term of the overall thickness series:

$$
\frac{\partial M_{r s}}{\partial T_{k}^{i}}=\frac{\partial M_{r s}}{\partial \bar{T}_{k}}
$$

Differentiating Eq.(6) for any specific $k$ and $i$ gives:

$$
\frac{\partial M_{r s}}{\partial T_{k}^{i}}=\rho_{m} \sum_{i=1}^{3} \sum_{j=1}^{6} \sum_{i=1}^{3} \sum_{i=1}^{6} U_{i} V_{j} U_{i l} V_{i j} \iint_{\text {area }} x^{m} y^{n} d x d y
$$

where

$$
\begin{aligned}
& m=m_{j}^{v}+m_{j}^{v}+m_{r}^{w}+m_{s}^{w}+m_{k}^{t} \\
& n=n_{i}^{u}+n_{\|}^{u}+n_{j}^{v}+n_{j}^{v}+n_{r}^{w}+n_{s}^{w}+n_{k}^{t}
\end{aligned}
$$

2) Mass matrix sensitivities with respect to shape design variables

The mass matrix is dependent on the shape variables through the coefficients $U_{1}$ and $V_{j}$ and the area integrals $I_{R T}(m, n)$. Analytical sensitivities for $U_{i}$ and $V_{j}$ are obtained through direct differentiation of equation (11) of part one of the paper. The derivatives of the area integrals with respect to $x$, where $x$ is any shape variable, are to be prepared. With this information, the derivative of the mass term $M_{r s}$ is calculated as follows:

$$
\frac{\partial \mathrm{M}_{\mathrm{rs}}}{\partial \mathrm{X}}=\rho_{\mathrm{m}} \sum_{\mathrm{k}=1}^{\mathrm{N}_{\mathrm{H}}} \overline{\mathrm{T}}_{\mathrm{k}} \sum_{\mathrm{k}=1}^{3} \sum_{\mathrm{j}=1}^{6} \sum_{\mathrm{i}=1}^{3} \sum_{\mathrm{j}=1}^{6}\left\{\mathrm{U}_{\mathrm{i}} \mathrm{V}_{\mathrm{j}} \mathrm{U}_{\mathrm{li}} \mathrm{V}_{\mathrm{j}} \frac{\partial \mathrm{I}_{\mathbb{R}}}{\partial \mathrm{X}}+\frac{\partial\left(\mathrm{U}_{\mathrm{i}} \mathrm{V}_{\mathrm{j}} \mathrm{U}_{\mathrm{i}} \mathrm{V}_{\mathrm{j}} \mathrm{O}\right.}{\partial \mathrm{X}} \mathrm{I}_{\mathbb{R}}\right.
$$




$$
\begin{aligned}
& \frac{\partial Q_{\text {stiff }_{n}}}{\partial x}=\cos \phi \sum_{i=1}^{3} \sum_{j=1}^{6} \sum_{i=1}^{3} \sum_{i=1}^{6}\left\{\left.\frac{\partial\left(U_{i} V_{j} U_{i i} V_{i j}\right)}{\partial x}\left(m_{i j}^{v}+m_{s}^{w}\right)\right|_{\operatorname{Tr}(m-1, n)}+\right. \\
& \left.U_{i} V_{j} U_{i i} V_{i j}\left(m_{i j}^{v}+m_{s}^{w}\right) \frac{\partial I_{T R(m-1, n)}}{\partial x}\right\}+ \\
& \sin \phi \sum_{\mathrm{i}=1}^{3} \sum_{\mathrm{j}=1}^{6} \sum_{\mathrm{i}=1}^{3} \sum_{\mathrm{ij=1}}^{6}\left\{\left.\frac{\partial\left(\mathrm{U}_{\mathrm{i}} \mathrm{V}_{\mathrm{j}} \mathrm{U}_{\mathrm{ii}} \mathrm{V}_{\mathrm{ij}}\right)}{\partial \mathrm{x}}\left(\mathrm{n}_{\mathrm{ii}}^{\mathrm{u}}+n_{\mathrm{j}}^{\mathrm{v}}+\mathrm{n}_{\mathrm{s}}^{\mathrm{w}}\right)\right|_{\mathrm{TR}(m, n-1)}+\right. \\
& \left.U_{i} V_{i} U_{i i} V_{i j}\left(n_{i j}^{u}+n_{i j}^{v}+n_{s}^{w}\right) \frac{\partial I_{T R(m, n-1)}}{\partial x}\right\}
\end{aligned}
$$

\section{Aerodynamic matrix shape sensitivities}

Shape derivatives of the aerodynamic damping and stiffness matrices are obtained by explicitly differentiating Eqs.(23) and (24):

$$
\frac{\partial Q_{\text {damp }}}{\partial x}=\sum_{i=1}^{3} \sum_{j=1}^{6} \sum_{i=1}^{3} \sum_{i=1}^{6}\left\{\left.\frac{\partial\left(U_{i} V_{j} U_{i i} V_{i j}\right)}{\partial x}\right|_{T R(m, n)}+U_{i} V_{j} U_{i i} V_{i j} \frac{\left.\partial\right|_{T R(m, n-1)}}{\partial x}\right\}
$$

where the powers $\mathrm{m}$ and $\mathrm{n}$ are given by Eq.(25).

Recall from Eqs.(26) that the coefficients relating $\left[A_{\text {stiff }}\right]$ and $\left[A_{\text {damp }}\right]$ to $\left[Q_{\text {stiff }}\right]$ and $\left[Q_{\text {damp }}\right]$ are functions of the Mach number only. Therefore, the sensitivities of $\left[A_{\text {stiff }}\right]$ and $\left[\mathrm{A}_{\text {damp }}\right]$ are:

$$
\begin{aligned}
& \frac{\partial A_{\text {stiff }}}{\partial D V}=\frac{1}{\sqrt{M_{\infty}^{2}-1}} \cdot \frac{\partial Q_{\text {stiff }}}{\partial D V} \\
& \frac{\partial A_{\text {damp }}}{\partial D V}=\frac{1}{\sqrt{M_{\infty}^{2}-1}} \cdot \frac{M_{\infty}^{2}-2}{M_{\infty}^{2}-1} \cdot \frac{\partial Q_{\text {damp }}}{\partial D V}
\end{aligned}
$$

Since $\left[Q_{\text {stiff }}\right]$ and $\left[Q_{\text {damp }}\right]$ are not affected by the Mach number, it is straightforward to obtain sensitivities with respect to Mach number as follows:

$$
\frac{\partial A_{\text {stiff }}}{\partial M_{\infty}}=\frac{\partial\left(\frac{1}{\sqrt{M_{\infty}^{2}-1}}\right)}{\partial M_{\infty}} \cdot Q_{\text {stiff }}
$$




$$
\frac{\partial A_{\text {damp }}}{\partial M_{\infty}}=\frac{\partial\left(\frac{1}{\sqrt{M_{\infty}^{2}-1}} \cdot \frac{M_{\infty}^{2}-2}{M_{\infty}^{2}-1}\right)}{\partial M_{\infty}} \cdot Q_{\text {damp }}
$$

\section{Flutter eigen value sensitivities}

The eigen vectors $\{\phi\}$ are defined in Eq.(33), and $\{\psi\}$ are obtained from the adjoin eigen value problem:

$$
\{\psi\}^{\top}[\bar{U}]=\lambda\{\psi\}^{\top}[\bar{V}]
$$

Differentiating Eq.(33) with respect to any design variable and pre-multiplying by the left eigen vectors, leads to the derivative of an eigen value with respect to any design variable:

$$
\frac{\partial \lambda}{\partial \mathrm{DV}}=\frac{\{\psi\}^{\top}\left[\frac{\partial[\overline{\mathrm{U}}]}{\partial \mathrm{DV}}-\lambda \frac{\partial[\overline{\mathrm{V}}]}{\partial \mathrm{DV}}\right]\{\phi\}}{\{\psi\}^{t}[\overline{\mathrm{V}}]\{\phi)}
$$

where the derivatives of the system matrices are obtained from:

$$
\begin{aligned}
& \frac{\partial[\overline{\mathrm{U}}]}{\partial \mathrm{DV}}=\left[\begin{array}{cc}
{[0]} & {[0]} \\
-\frac{\partial[\overline{\mathrm{K}}]}{\partial \mathrm{DV}} & -\frac{\partial[\mathrm{C}]}{\partial \mathrm{DV}}
\end{array}\right] \\
& \frac{\partial[\overline{\mathrm{V}}]}{\partial \mathrm{DV}}=\left[\begin{array}{ll}
{[0]} & {[0]} \\
{[0]} & \frac{\partial[\mathrm{M}]}{\partial \mathrm{DV}}
\end{array}\right] \\
& \frac{\partial[\mathrm{C}]}{\partial \mathrm{DV}}=-\rho_{\infty} \mathrm{U}_{\infty} \frac{\partial\left[\mathrm{A}_{\text {damp }}\right]}{\partial \mathrm{DV}} \\
& \frac{\partial[\overline{\mathrm{K}}]}{\partial \mathrm{DV}}=\frac{\partial[\mathrm{K}]}{\partial \mathrm{DV}}+\frac{\partial\left[\mathrm{K}_{\mathrm{G}}\right]}{\partial \mathrm{DV}}-\rho_{\infty} \mathrm{U}_{\infty}^{2} \frac{\partial\left[\mathrm{A}_{\text {stif }}\right]}{\partial \mathrm{DV}}
\end{aligned}
$$

In panel flutter optimization studies it has been common to use a flutter dynamic pressure constraint in search of an optimal panel with a given critical dynamic pressure. Let the real and imaginary parts of the aeroelastic poles be given by: 


$$
\lambda=\sigma+\mathrm{j} \omega
$$

The real part $\sigma$ and the imaginary part $\omega$ are functions of dynamic pressure $q_{D}$, Mach number, and any sizing or shape design variables. At a given Mach number $\sigma=\sigma$ ( $\left.q_{D}, D V\right)$ and at the flutter dynamic pressure $\sigma=\sigma\left(q_{\text {filuter }}(D V)\right.$, DV), defined at whatever value of damping used to determine flutter. Differentiating with respect to DV and setting the derivative to zero to enforce the flutter criterion:

$$
\frac{\partial \sigma}{\partial \mathrm{DV}_{\text {(at flutter) }}}=\frac{\partial \sigma}{\partial \mathrm{DV}}+\frac{\partial \sigma}{\partial \mathrm{q}_{\text {flutter }}} \cdot \frac{\partial \mathrm{q}_{\text {flutter }}}{\partial \mathrm{DV}}=0
$$

Thus,

$$
\frac{\partial \mathrm{q}_{\text {fluter }}}{\partial \mathrm{DV}}=-\frac{\left(\frac{\partial \sigma}{\partial \mathrm{DV}}\right)}{\left(\frac{\partial \sigma}{\partial \mathrm{q}_{\text {flutter }}}\right)}=-\frac{\operatorname{Re}\left(\frac{\partial \lambda}{\partial \mathrm{DV}}\right)}{\operatorname{Re}\left(\frac{\partial \lambda}{\partial \mathrm{q}_{\text {flutter }}}\right)}
$$

The numerator is already known from Eq.(46). To obtain the denominator, Eq.(33) is differentiated with respect to dynamic pressure at the flutter dynamic pressure:

$$
\frac{\partial \lambda}{\partial \mathrm{q}_{\mathrm{D} \text { (at flutter })}}=\frac{\{\psi\}^{\mathrm{T}}\left[\frac{\partial[\overline{\mathrm{U}}]}{\partial \mathrm{q}_{\mathrm{D}}}-\lambda \frac{\partial[\overline{\mathrm{V}}]}{\partial \mathrm{DV}}\right]\{\phi\}}{\{\psi\}^{\mathrm{T}}[\overline{\mathrm{V}}]\{\phi\}}
$$

\section{TEST CASES AND RESULTS}

Before proceeding to study analytic sensitivities and approximations using the present capability, it is important to assess the accuracy of its panel flutter results. For this, results of the present analysis technique for simply supported panels have been compared to reported results using other solution techniques. Correlation with the results of [14] are shown to demonstrate the capability of the present technique to calculate flutter dynamic pressures accurately for skewed, composite simply supported panels.

The simply supported panels studied in [14] are skewed with skew angles varying from 0 to 45 degrees. A unidirectional fiber composite material is used with fiber angle varying from 0 to 90 degrees. Sides of the panels are equal in length. Material properties are $E_{1}=137 \times 10^{9} \mathrm{~Pa}, \mathrm{E}_{2}=9.7 \times 10^{9} \mathrm{~Pa}, \mathrm{G}_{12}=5.5 \times 10^{9} \mathrm{~Pa}, v_{12}=0.3$ and $\rho_{\mathrm{m}}$ $=1580 \mathrm{~kg} / \mathrm{m}^{3}$. Critical dynamic pressure and frequency are nondimensionalized as: 


$$
\begin{aligned}
& \Lambda_{\text {crit }}=\frac{2 \mathrm{q}_{D_{\text {ett }}} a^{3}}{E_{2} h^{3} \sqrt{M_{\infty}^{2}-1}} \\
& \Omega_{\text {crit }}=\omega_{\text {crit }} a^{2} \sqrt{\frac{\rho_{m}}{E_{2} h^{2}}}
\end{aligned}
$$

where $a$ is the side of panel and $h$ its thickness. Both critical and flutter dynamic pressures have been calculated and the comparison of critical dynamic pressure and critical frequency with the results of [14] are shown in Fig.1 and Fig.2. As the figures show, the correlation is good over a considerable range of skew and fiber angles, except for cases in which ske,w angles and fiber angles are simultaneously large. In the flutter analysis case discussed here, mass and aerodynamic matrices play an important role in addition to the stiffness and geometric stiffness matrices.

To assess the accuracy of the polynomial Ritz flutter analysis in cases involving inplane loading, results obtained with the present capability are compared with finite element results from [12]. Square all-aluminum simply supported panels are analyzed, subject to different in-plane loading. A nondimensional in-plane force is defined as:

$$
r_{i j}=\left(\mathrm{N}_{\mathrm{ij}} / \pi^{2}\right)\left(a^{2} / \mathrm{D}\right)
$$

and Table 1 summarizes the results, where the normalized critical dynamic pressure is defined by:

$$
\Lambda_{\text {crit }}=\frac{2 \mathrm{q}_{\mathrm{D}_{\text {ext }}} a^{3}}{D \sqrt{M_{\infty}^{2}-1}}
$$

The correlation is good for moderate in-plane loading. In the case of $\mathrm{N}_{\mathrm{xy}}$ loading (pure shear), the correlation becomes worse as intensity of in-plane loads increases.

\begin{tabular}{|c|c|c|c|}
\hline$r_{i y}$ & Ref.(12) & Present & \% difference \\
\hline$r_{x x}=-3$ & 275.7 & 265.1 & 3.85 \\
\hline$r_{x x}=0$ & 518.2 & 512.6 & 1.08 \\
\hline$r_{x x}=3$ & 789.0 & 793.1 & 0.50 \\
\hline$r_{x y}=2$ & 487.0 & 473.1 & 2.85 \\
\hline$r_{x y}=4$ & 418.9 & 381.7 & 8.90 \\
\hline$r_{x y}=6$ & 321 & 274.3 & 14.7 \\
\hline
\end{tabular}

Table 1: Normalized critical dynamic pressures for aluminum square panels under in-plane loads 

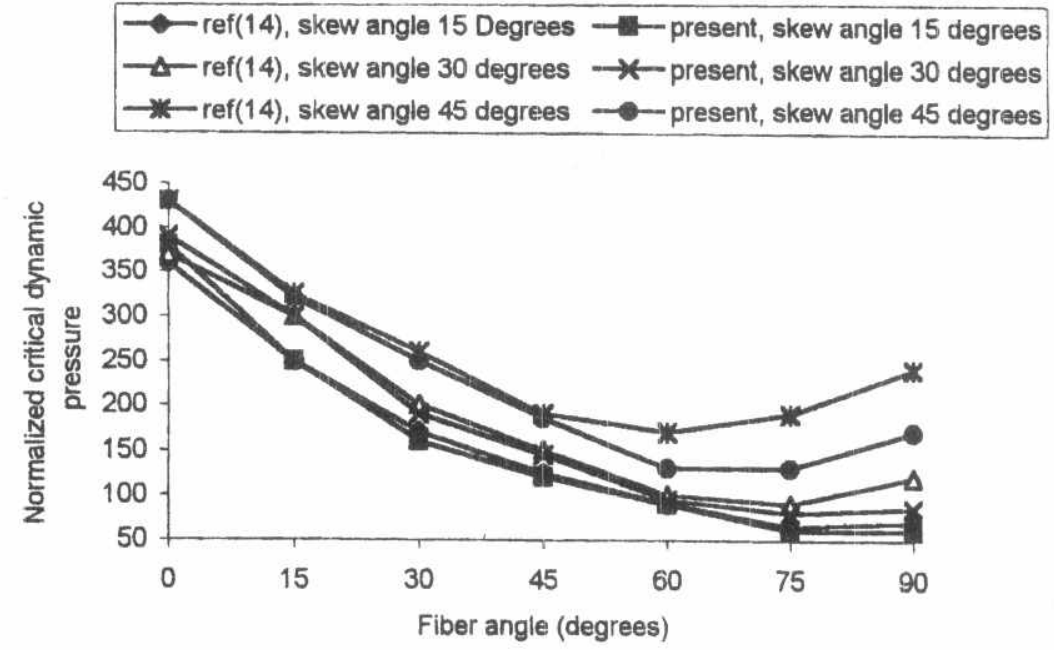

Fig.1: Normalized critical dynamic pressure comparisons with ref(14) for a range of skew angles and fiber orientation angles

- ref(14), skew angle 15 degrees - - present, skew angle 15 degrees
$-\Delta-$ ref(14), skew angle 30 degrees - - present, skew angle 30 degrees
$-\ldots$ - ref(14), skew angle 45 degrees - - present, skew angie 45 dgrees

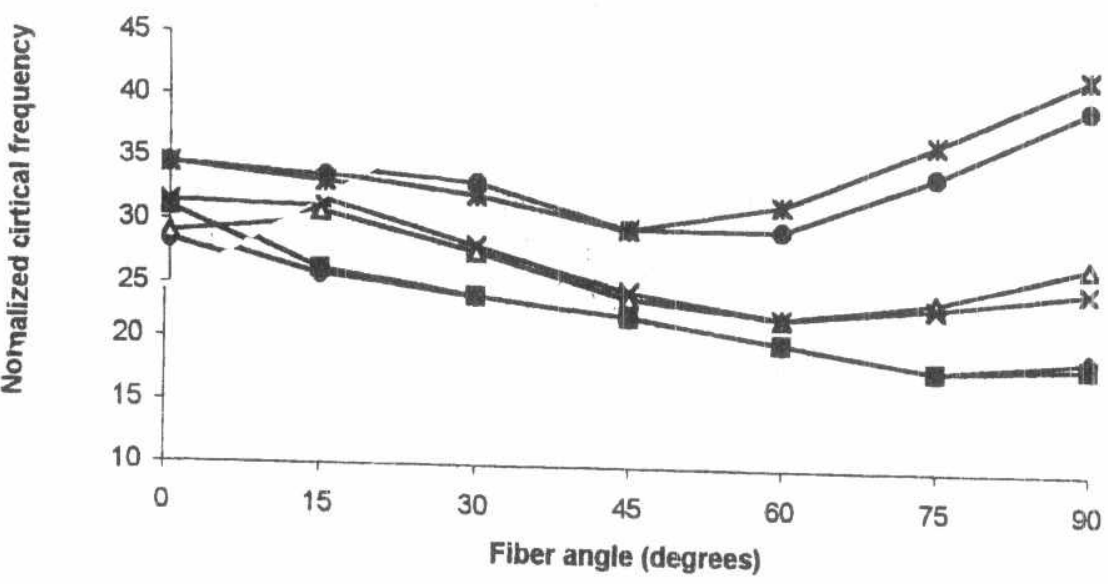

Fig.2: Normalized critical frequency comparisons with ref(14) for a range of skew angles and fiber orientation angles 


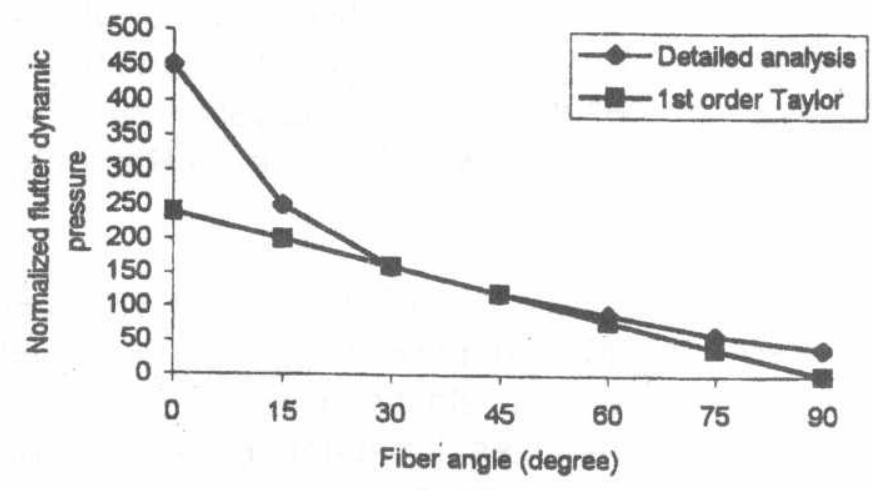

Fig.3: Detailed analysis and first order Taylor series approximation for normalized flutter dynamic pressure for a skewed composite panel. Skew angle 15 degrees

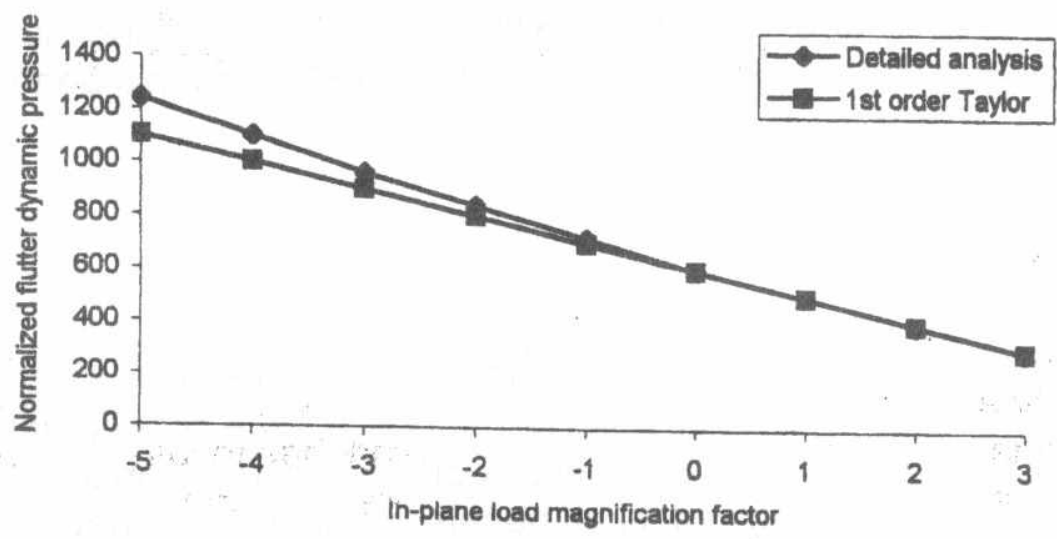

Fig.4: Linear Taylor series approiximation of panel flutter dynamic pressure for a 30 degrees skewed panel, subject to a varying system of in-plane loads 
With analytic derivatives available, it is possible to assess the accuracy of finite difference derivatives and examine the range of acceptable step size. As has been shown for the case of polynomial Ritz analysis of wing box structures,[19], ill conditioning (when high-order polynomials are used) can lead to erroneous finite difference derivatives, even in cases when the analysis itself is adequate. In panel flutter analysis cases studied here, and with the order of Ritz polynomials used, no ill-conditioning problems were encountered.

Finite difference derivatives were used to verify the analytic derivatives for a variety of cases including different design variables (thickness, material and shape) and different behavior functions (such as aeroelastic poles, or critical and flutter dynamic pressures). Using the analytic derivatives, it became possible to examine first-order Taylor series approximations in various cases and to gain some experience as to the move limits that might be required. In Fig.3, the analytic sensitivity of flutter dynamic pressure with respect to fiber angle is used to construct first-order approximation for a 15 degree skewed panel. In Fig.4, a first-order Taylor approximation is constructed based on the analytic sensitivity of flutter dynamic pressure with respect to a magnification factor applied to the system of combined in-plane loads on a skewed panel. An isotropic panel with a skew angle of 30 degrees, equal sides of $1(\mathrm{~m})$ and thickness of $3(\mathrm{~mm})$ is subjected to $N_{x}=-2000(\mathrm{~N} / \mathrm{m})$ and $N_{y}=-1000(\mathrm{~N} / \mathrm{m})$. The geometric stiffness matrix corresponding to this in-plane loading is $\left[K_{G}\right]_{r e f}$ and the inplane loads are varied so that the actual geometric stiffness matrix $\left[K_{G}\right]=\eta\left[K_{G}\right]_{\text {ref }}(\eta$ is a multiplication factor increasing or decreasing a system of in-plane loads). It is easy to obtain the derivative of $\left[K_{G}\right]$ with respect to $\eta$ in this case, and the results simulate a situation where aeroelastic stability of a skin panel is influenced by changes in load distribution in the larger structure containing this panel.

\section{CONCLUSIONS}

The capability to include configuration shape-design variables, in any multidisciplinary design optimization of airplanes in the conceptual or prelimirary design stages, is essential. The present work has focused on the design-orierited aeroelastic analysis of optimized skin panels in supersonic flow. Since, in typical optimum aeroelastic synthesis of wing structures, many skin panels can be buckling critical, and since stressing a panel up to a point close to its buckling load may have considerable effect on its aeroelastic stability, it becomes important to develop efficient analysis and sensitivity capabilities for panel flutter constrairits.

It is shown in this work how modeling and Ritz formulation based on simple polynomial functions in global coordinates lead to the efficient evaluation of panel stiffness, geometric stiffness, and mass, as well as aerodynamic damping and aerodynamic stiffness matrices. Using analytic formulas for area integrals of polynomial terms over general trapezoidal area shapes, it is shown that no 
numerical integration is needed for evaluating panel structural or aerodynamic matrices. A table of area integrals for the panel needs to be evaluated only once for a given panel shape,[19]. Then, structural and aerodynamic matrices, as well as their analytic sensitivities with respect to sizing, material, and shape design variables, can be obtained by linear combinations of members of this table of integrals.

Systematic evaluation of the resulting panel flutter prediction capability was carried out, comparing results from the present work with results from other references. Overall, the current capability led to good correlation with other prediction techniques up to panel leading-edge sweep angles of $\mathbf{3 0}$ degrees.

Expressions for analytic sensitivity of panel aeroelastic poles and resulting flutter dynamic pressure have been obtained and checked against finite difference sensitivities. Excellent correlation and a wide range of step sizes adequate for the finite difference derivatives has been found. Used, in turn, in Taylor series approximations for the flutter dynamic pressure, the flutter and sensitivity results have been shown to lead to robust approximations over a wide range of design variable changes. The work has also shown how to integrate the panel aeroelastic analysis and sensitivity predictions with a wing box analysis and sensitivity capability, where in-plane loads determined by the wing box behavior serve as inputs to the panel aeroelastic behavior.

\section{REFERENCES}

[1] Dugundji, J., "Theoretical Consideration of Panel Flutter at High Supersonic Mach Numbers", AIAA Journal, Vol.4, No.7, pp 1257-1266,(1966)

[2] Eastep,F.E., and Mclntosh,S.C., "Analysis of Nonlinear Panel Flutter and Response Under Random Excitation or Nonlinear Aerodynamic Loading", AIAA Journal, Vol.9, No.3, pp 411-418, (1971)

[3] Sander,G., Bon,C., and Gerandin,M., "Finite Element Analysis of Supersonic Panel Flutter", Int.J. for Numerical Methods in Engineering, Vol.7, pp 379-397, (1973)

[4] Yang,T.Y., "Flutter of Flat Finite Element Panels in Supersonic Unsteady Potential Flow", AIAA Journal, Vol.13, No.11, pp 1502-1507, (1975)

[5] Bismarck-Nasr,M.N., "Finite Element Analysis of Aeroelasticity of Plates and Shells", Applied Mechanics Review, Vol.45, No.12, pp 461-482, (1992)

[6] Ibrahim,R.A., Orono,P.O., and Madaboosi,S.R., "Stochastic Flutter of a Panel Subjected to Random In-plane Forces, Part I: Two mode Interaction", AIAA Journal, Vol.28, No.4, pp 694-702, (1990)

[7] Liaw,D.G., "Supersonic Flutter of Laminated Thin Plates with Thermal Effects", J. of Aircraft, Vol.30, No.1, pp 105-111, (1993)

[8] Shore,C.P., "Flutter Design Charts for Biaxially Loaded Isotropic Panels", J. of Aircraft, Vol.7, No.4, pp 325-329, (1970) 
[9] Lin, K.L., Lu,P.J., and Tarn,J.O., "Flutter Analysis of Composite Panels Using High Precision Finite Elements", Computers and Structures, Vol.33, No.2., pp 561-574, (1989)

[10] Chowdary, T.V.R., Parthen,S., and Sinha,P.K., "Finite Element Flutter A.nalysis of Laminated Composite Panels", Computers and Structures, Vol. 53, No.2., pp 245-251,(1994)

[11] Lee,I., and Cho,M.H., "Supersonic Flutter Analysis of Clamped Composite Panels Using Shear Deformable Finite Elements", AIAA Journal, Vol.29, No.5, pp 782-783, (1991)

[12.] Kariapp, Somashekar,B.R., and Shah,C.G., "Discrete Element Approach to Flutter of Skew Panels with In-plane Forces Under Yawed Supersonic Flow", AIAA Journal, Vol.8, No.11,. pp 2017-2022,(1970)

[13] Srinivasan,R.S., and Babu,J.C., "Free Vibration and Flutter of Laminated Quadrilateral Plates," Computers and Structures, Vol.27, No.2. pp 297-403, (1987)

[14] Chowdary, T.V.R., Sinha, P.K., and Parthan,S., "Finite Element Flutter Analysis of Composite Skew Panels", Computers and Structures, Vol.58, No.3, pp 613620, (1996)

[15] Weisshaar, T.A., "Aeroelastic Optimization of a Panel in High Mach Number Supersonic Flow", J. of Aircraft, Vol.9, No.9, pp 611-617, (1972)

[16] Weisshaar,T.A., "Panel Flutter Optimization - A Refined Finite Element Approach", Int. J. for Numerical Methods in Engineering, Vol.10, No.1, pp 7791, (1976)

[17] Van Keuren,G.M., and Eastep,F., "Use of Galerkin's Method for Minimurn Weight Panels with Dynamic Constraints", J. of Spacecraft and Rockets, Vol.14, No.7, pp 414-418, (1977)

[18] Librescu, L., and Beiner,L., "Weight Minimization of Orthotropic Flat Panels Subjected to a Flutter Speed Constraint", AIAA Journal, Vol.24, no.6, pp 981997, (1986)

[19] Livne,E., "Analytical Sensitivities for Shape Optimization in Equivalent Plate Structural Wing Models", J. of Aircraft, Vol.31, No.4, pp 961-969, (1994)

[20] Livne,E., and Li,W.L., "Aeroservoelastic Aspect of Wing/Control Surface Planform Shape optimization", AIAA Journal, Vol.33, No.2, pp 302-311, (1995)

[21] Voss, H.M., and Dowell,E.H., "Effect of Aerodynamic Damping on Flutter of Thin Plates", AIAA Journal, Vol.2, No.1, pp 119-120, (1964)

[22.] Dowell,E.H., "Aeroelasticity of Plates and Shells", Noordhoff International, Leyden, The Netherlands, 1975. 